\title{
RISCO DE INTEGRIDADE DA PELE PREJUDICADA EM IDOSOS HOSPITALIZADOS
}

\author{
Suelen Gomes Malaquias ${ }^{1}$, Maria Márcia Bachion², Adélia Yaeko Kyosen Nakatani ${ }^{3}$
}

\begin{abstract}
RESUMO: A higidez da pele é fundamental para a manutenção da saúde humana. Com o envelhecimento e a vigência de internação hospitalar, o idoso encontra-se exposto ao "Risco de integridade da pele prejudicada". Este estudo objetivou analisar a ocorrência do diagnóstico de Enfermagem "Risco de integridade da pele prejudicada” em idosos hospitalizados. Trata-se de estudo descritivo, realizado em um hospital-escola de Goiânia, estado de Goiás, de maio a junho de 2007. Para a coleta de dados foi utilizado um instrumento contendo dados sóciodemográficos e clínicos relativos aos fatores de risco do Diagnóstico de Enfermagem (DE) estudado. Participaram 23 idosos, sendo 56,52\% do sexo feminino, na faixa etária entre 70 e 79 anos (60,87\%). Foi identificado o DE estudado em 100\% dos participantes. Encontrou-se fatores de risco que se configuram em alterações decorrentes do processo de envelhecimento, de exposição a ações ou eventos extrínsecos, estilo de vida e comportamentos em saúde.
\end{abstract}

PALAVRAS-CHAVE: Idoso; Enfermagem; Diagnóstico de enfermagem; Pele.

\section{RISK OF IMPAIRED SKIN INTEGRITY IN HOSPITALIZED ELDERLY}

ABSTRACT: Skin health is essential to the maintenance of human health. With aging, frequent hospitalizations, elderly people are exposed to the "Risk of impaired skin integrity”. Objective: This paper aimed at analyzing the occurrence of this nursing diagnosis in hospitalized elderly patients. It is a descriptive study carried out in a teaching-hospital in Goiânia, state of Goiás, from May to June/2007. Data collection was held by means of an instrument which used clinical and sociodemographic data related to the risk factors for the studied Nursing Diagnosis (ND). A total of 23 elderly subjects took part in this study, $56.52 \%$ were women, ranging from 70 to 79 years of age $(60.87 \%)$. The nursing diagnosis studied in $100 \%$ of the subjects was identified. Risk factors were found involving changes due to aging process, exposure to extrinsic factors or events, lifestyle and health behaviors.

KEYWORDS: Aged; Nursing; Nursing diagnosis; Skin.

\section{RIESGO DE LA INTEGRIDAD DE LA PIEL PERJUDICADA EN PERSONAS MAYORES HOSPITALIZADAS}

RESUMO: El bienestar de la piel es fundamental para el mantenimiento de la salud humana. Con el envejecimiento y el período de internación hospitalar, las personas mayores se encuentran expuestas al "riesgo de tener la integridad de la piel perjudicada”. Objetivo: Este estudio tuvo por objetivo analizar como ocurre este diagnóstico, en personas mayores hospitalizadas. Método: Se trata de un estudio descriptivo, realizado en un hospital-escuela de Goiânia, estado de Goiás, de mayo hasta junio del 2007. Para la recolección de datos, fue utilizada una encuesta, conteniendo datos sociodemográficos y clínicos relativos a los factores de riesgo del DE estudiado. Resultados: Tuvimos la participación de 23 personas mayores, de las cuales 56,52\% eran del sexo femenino, con edad entre 70 y 79 años (60,87\%). Fue identificado el DE estudiado, en el 100\% de los participantes. Conclusión: Fueron encontrados factores de riesgo que comprenden alteraciones decorrentes del proceso de envejecimiento, por estar expuestos a acciones o actividades intrínsecas, estilo de vida y la importancia que le dan a la salud.

PALABRAS CLAVE: Anciano; Enfermería; Diagnóstico de enfermería; Piel.

${ }^{1}$ Enfermeira. Especialista em Enfermagem Dermatológica. Mestranda do Programa de Pós-Graduação-Mestrado em Enfermagem da Faculdade de Enfermagem da Universidade Federal de Goiás-FEN/UFG. Professor Substituto da FEN/UFG.

${ }^{2}$ Enfermeira. Doutora em Enfermagem. Professor Titular da FEN/UFG. Orientadora.

${ }^{3}$ Enfermeira. Doutora em Enfermagem. Professor Adjunto da FEN/UFG.

Autor correspondente:

Suelen Gomes Malaquias

Rua 8, 39 - 74063-150 - Goiânia-GO

Recebido: 02/11/07

E-mail: sgmalaquias@yahoo.com.br

Aprovado: 18/04/08

Cogitare Enferm 2008 Jul/Set; 13(3):428-36 


\section{INTRODUÇÃO}

A pele é um órgão que desempenha diversas funções, entre as quais a de proteção, o que resguarda o organismo da agressão de diversos fatores, dentre eles: os agentes mecânicos, físicos e biológicos por meio de células especializadas e componentes protéicos que constituem a organização cutânea ${ }^{(1)}$. O processo de envelhecimento desencadeia diversas alterações na pele, as quais sobrevêm por motivos como: a redução da elasticidade, menor percepção da temperatura ambiente e suas variações, diminuição da sensibilidade tátil, bem como do componente aquoso ${ }^{(2)}$.

As alterações funcionais, decorrentes do envelhecimento, predispõem o indivíduo à ocorrência de doenças crônicas não transmissíveis, as quais podem desencadear algumas condições que levam às hospitalizações. As admissões e readmissões hospitalares de idosos podem, também, estar associadas às condições precárias de vida e à baixa renda ${ }^{(3)}$.

Frente ao exposto, pode-se depreender que, no contexto hospitalar, os idosos podem estar em risco de desenvolverem lesões cutâneas devido a múltiplos fatores e, a enfermagem deve estar atenta às condições da pele destas pessoas. Esta profissão, em sua história, busca a organização do cuidado, com a incorporação do método científico, por meio do Processo de Enfermagem, visando qualidade no atendimento às pessoas. Nesse sentido, a avaliação das condições de saúde, a qual inclui o exame da pele e identificação da presença de riscos à sua integridade, consta da primeira etapa do Processo de Enfermagem (PE), denominada coleta de dados. A partir do julgamento clínico destas informações, chega-se à segunda etapa, o Diagnóstico de Enfermagem (DE) ${ }^{(4)}$.

Para afirmação da inferência diagnóstica, a Enfermagem dispõe de diferentes taxonomias, entre as quais, a da North American Nursing Diagnosis Association (NANDA) ${ }^{(5)}$, que define Risco de Integridade da pele prejudicada como a condição de um indivíduo "estar em risco da pele ser alterada de forma adversa" por diversos fatores externos: radiação, imobilização física, fatores mecânicos, hipotermia ou hipertermia, umidade, substância química, excreções e/ ou secreções, pele úmida e extremos de idade; ou, ainda: medicamentos, proeminências ósseas, fatores imunológicos, fatores de desenvolvimento, sensibilidade e/ou pigmentação alteradas, alteração metabólica, circulação alterada, alterações no turgor da pele e no estado nutricional, bem como fatores psicogênicos ${ }^{(5)}$.
Uma condição inicial de risco de integridade de pele prejudicada pode evoluir para múltiplas situações de respostas humanas, como risco de infecção, dor aguda e dor crônica, baixa autoestima situacional, medo, ansiedade, entre outros ${ }^{(5)}$. Neste contexto, manter a higidez da pele mediante ações de promoção de saúde e detecção precoce da presença do risco de lesão deve ser uma preocupação constante da enfermagem, no ambiente hospitalar.

Estudos, especificamente, sobre a identificação de "risco de integridade da pele prejudicada", em idosos ainda são escassos. Não encontramos pesquisas envolvendo idosos hospitalizados, em revisão das bases MEDLINE (Literatura Internacional em Ciências da Saúde) e NLM (National Library of Medicine).

Assim, com a realização deste estudo, esperamos contribuir para estabelecer bases para o planejamento da assistência de enfermagem, favorecendo a qualidade do atendimento à saúde dos idosos, no contexto da hospitalização, além de desenvolver conhecimento sobre a ocorrência desse evento, na população estudada, sob o enfoque da enfermagem gerontológica.

Constituiu objetivo geral desta pesquisa: analisar a ocorrência de risco para integridade da pele prejudicada em idosos hospitalizados. Objetivos específicos: caracterizar os principais aspectos sóciodemográficos e clínicos dos idosos participantes do estudo, identificar a ocorrência deste diagnóstico em idosos hospitalizados e, verificar os fatores de risco para este diagnóstico na população estudada.

\section{DESCRIÇÃO DO MÉTODO}

Este estudo descritivo, com abordagem quantitativa, foi realizado de maio a junho de 2007, em um hospital-escola de Goiânia-Goiás, nas Clínicas Médica, Cirúrgica e de Patologias Tropicais. O projeto desta pesquisa foi aprovado pelo Comitê de Ética em Pesquisa Médica Humana e Animal do Hospital das Clínicas/UFG (protocolo no . 62/2007).

Foram estabelecidos como critérios de inclusão de idosos: estarem conscientes, orientados auto e alo psiquicamente, em condições clínicas favoráveis, demonstrarem desejo expresso em participar da pesquisa, manifestado pela assinatura do Termo de Consentimento Livre e Esclarecido. Os critérios de exclusão foram: estarem desorientados, em estado grave, saírem de alta antes do término da coleta de dados, ou ainda, se solicitar a retirada de sua participação. 
Do total de 86 idosos que estiveram internados no período, 11 apresentaram estado de desorientação, 29 estavam no período pós-operatório imediato e três em estado grave no momento da abordagem inicial do convite para participarem do estudo, sendo estes excluídos. Dos 43 elegíveis, 14 receberam alta antes de serem abordados para a pesquisa. Assim, foram convidados vinte e nove idosos ( 60 anos ou mais), sendo que 23 participaram e seis recusaram.

Os idosos foram avaliados mediante um instrumento de coleta de dados que continha duas partes: a primeira com dados sóciodemográficos (idade, sexo, estado civil, escolaridade, renda, ocupação) e internação atual (motivo da internação, diagnóstico médico, número de internações anteriores); a segunda compreendia informações da avaliação do "Risco de Integridade da pele prejudicada", por meio de exame dos fatores de risco conforme NANDA(5), e de avaliação de risco para desenvolvimento de úlceras por pressão (UP) com a Escala de Waterlow ${ }^{(6)}$. Esta escala considera: constituição, peso para altura, continência, tipo de pele ou área de risco visível, sexo, idade, mobilidade, apetite, má nutrição dos tecidos, déficit neurológico, cirurgia de grande porte, trauma e uso de determinados medicamentos. A pontuação da somatória de cada parâmetro classifica o grau de risco como "de risco" (entre 10 e 14), "alto risco" (15 a 19) e "risco muito alto" (acima de 20) ${ }^{(6)}$. A avaliação compôs-se de um a dois encontros, com intervalo de 6 a 24 horas, com duração média de 60 minutos.

\section{RESULTADOS}

A idade dos idosos variou entre 60 e 87 anos, predominando os de faixa etária entre 70 e 79 anos (60,87\%). Houve leve predomínio do sexo feminino (56,52\%). Quanto ao estado civil, 11 (47,83\%) eram casados ou estavam em união consensual, quatro (17,39\%) solteiros ou desquitados e oito (34,78\%) viúvos.

A população idosa brasileira, pelo Censo de 2000, representava 8,6\% da população geral, sendo que 4,9\% estavam entre 60 e 69 anos e 3,7\% tinham 70 anos ou mais $^{(7)}$. Neste levantamento, os idosos encontravam-se em seus domicílios, diferente da população abordada neste estudo, em que, como em outros ${ }^{(3-4)}$, a faixa etária predominante é de 70 anos ou mais.

O número de mulheres participantes foi $13,42 \%$ superior ao dos homens. Isso mostra um perfil compatível com os dados da população idosa brasileira. De acordo com o Censo de 2000, a proporção de mulheres corresponde a 55,1\% dos idosos brasileiros ${ }^{(7)}$. A instituição onde a pesquisa foi realizada é referência para o Estado de Goiás. Dezessete (73\%) idosos eram do interior, sendo apenas seis $(26,1 \%)$ procedentes da cidade Goiânia. Este fato denota a escassez e/ou deficiência dos serviços de saúde que oferecem atendimento a esta população, nas diversas regiões de Goiás, em especial no entorno de Goiânia. Isto é preocupante, frente ao aumento acentuado do número de idosos nos últimos 20 anos, com demanda crescente da população nos serviços de saúde ${ }^{(7-8)}$.

Todos eram aposentados, sendo oito $(34,8 \%)$ ex-lavradores ou trabalhadores rurais. Quanto à escolaridade dos idosos, houve predominância de nãoalfabetizados $(52,17 \%)$, e com ensino fundamental incompleto (34,78\%). Os demais (13,05\%) apresentavam ensino fundamental completo. A escolaridade pode influenciar no acesso e/ou compreensão das informações relacionadas à saúde, de forma geral, e, de maneira especial, o autocuidado, visando manter a integridade da pele.

Somente um idoso $(4,3 \%)$ referiu renda familiar de três ou mais salários-mínimos, enquanto os demais $(95,7 \%)$ relataram receber de um a dois saláriosmínimos por mês.

Segundo o Censo brasileiro de $2000^{(7)}$, a região Centro-Oeste possuiu o segundo menor rendimento médio dos responsáveis idosos, por domicílios, seguida apenas da região Nordeste. A renda determina a capacidade do indivíduo em adquirir bens e serviços ${ }^{(9)}$. Neste sentido, o acesso a práticas fundamentais, para a determinação de uma vida saudável, pode estar comprometido na população idosa, podendo influenciar diretamente no processo de envelhecimento, tornandoa vulnerável na senescência.

Dezesseis idosos (69,56\%) referiram que a atual internação se configurava em readmissão, num período menor ou igual a um ano. Quatro idosos (17,40\%) relataram readmissão no período entre um e dez anos, dois $(8,70 \%)$ há mais de dez anos. Três (13,03\%) negaram hospitalização anterior.

Em um estudo realizado num hospital geral de Marília -SP, durante o período de um ano, houve índice de readmissões de pessoas idosas de $18 \%$ no período de um ano, o que remete a considerar este evento como de risco para a integridade cutânea, dentre outros, nesta população $0^{(3)}$.

Dentre os motivos de internações encontrados, as doenças cardiovasculares foram predominantes com $34,72 \%$, seguidas de neoplasias malignas (21,74\%). 
Estes resultados são semelhantes aos achados de outros estudos, como também em dados da população brasileira em geral, que mostram o aumento das taxas de mortalidade por doenças cardiovasculares e câncer à medida que a idade avança ${ }^{(9-10)}$.

Foi identificado o diagnóstico de enfermagem (DE), "risco de integridade da pele prejudicada", em $100 \%$ dos idosos participantes do estudo. O número de fatores de risco (FR) por idoso variou entre três e quinze, com média de 13, mediana de 12 e moda igual a 14 . Cinco FR foram identificados em todos os idosos participantes (100\%), dez foram encontrados entre 11 e 19 idosos (47,83 a 82,61\%), conforme Tabela 1.

Os fatores de risco "extremos de idade" e "fatores de desenvolvimento" estavam presentes em todos os participantes. Com o advento do processo de envelhecimento, alterações morfológicas, funcionais e bioquímicas tornam os indivíduos mais susceptíveis às agressões intrínsecas e extrínsecas. O fator de risco "radiação" foi encontrado em todos os idosos. O efeito da radiação sobre o homem contribui para maior incidência de neoplasias, dependendo da idade em que ocorreu a exposição, a quantidade recebida, o tempo e o número de exposições ${ }^{(11)}$.

Tabela 1 - Distribuição dos idosos ( $\mathrm{N}=23)$ segundo a presença de fatores de risco de integridade da pele prejudicada e pontuação para escala de Waterlow. Hospital-escola, Goiânia - Goiás, Mai/Jun, 2007

\begin{tabular}{|c|c|c|c|c|c|c|c|c|c|c|c|c|c|c|c|c|c|c|c|c|c|}
\hline \multirow[t]{2}{*}{ Fatores de risco } & \multicolumn{20}{|c|}{ Idosos Participantes } & \multirow[t]{2}{*}{ Total } \\
\hline & 1 & 2 & 3 & 4 & 5 & 6 & 78 & 89 & 10 & 11 & 121 & 1314 & 415 & 16 & 17 & 181 & 192 & 202 & 2122 & 2223 & \\
\hline Radiação & $\mathrm{x}$ & $\mathrm{x}$ & $\mathrm{X}$ & $\mathrm{x}$ & $\mathrm{x}$ & $\mathrm{x} 2$ & $\mathrm{x} \quad \mathrm{x}$ & $\mathrm{X} \quad \mathrm{X}$ & $\mathrm{x}$ & $\mathrm{x}$ & $\mathrm{x} \quad \mathrm{x}$ & $\mathrm{x} \quad \mathrm{X}$ & $x$ & $\mathrm{x}$ & $\mathrm{x}$ & $\mathrm{X} \quad \mathrm{x}$ & $\mathrm{x}$ & $\mathrm{x}$ & $\mathrm{X} \quad \mathrm{X}$ & $\mathrm{X} \quad \mathrm{X}$ & 23 \\
\hline Medicamentos & $\mathrm{x}$ & $\mathrm{x}$ & $\mathrm{x}$ & $\mathrm{x}$ & $\mathrm{x}$ & $\mathrm{x} 2$ & $\mathrm{x} \quad \mathrm{x}$ & $\mathrm{x} \quad \mathrm{X}$ & $\mathrm{x}$ & $\mathrm{x}$ & $\mathrm{x} \quad \mathrm{x}$ & $\mathrm{x} \quad \mathrm{X}$ & $x$ & $\mathrm{x}$ & $\mathrm{x}$ & $\mathrm{X} \quad \mathrm{x}$ & $\mathrm{x}$ & $\mathrm{x}$ & $\mathrm{x} \quad \mathrm{x}$ & $\mathrm{X} \quad \mathrm{X}$ & 23 \\
\hline Fatores imunológicos & $\mathrm{x}$ & $\mathrm{x}$ & $\mathrm{x}$ & $\mathrm{x}$ & $\mathrm{x}$ & $\mathrm{x} \quad 2$ & $\mathrm{x} \quad \mathrm{x}$ & $x \quad x$ & $\mathrm{x}$ & $\mathrm{x}$ & $\mathrm{x} \quad \mathrm{x}$ & $\mathrm{x} \quad \mathrm{x}$ & $x$ & $\mathrm{x}$ & $\mathrm{x}$ & $\mathrm{x} \quad \mathrm{x}$ & $\mathrm{x}$ & $\mathrm{x}$ & $\mathrm{x} \quad \mathrm{x}$ & $\mathrm{x} \quad \mathrm{x}$ & 23 \\
\hline Extremos de idade & $\mathrm{x}$ & $\mathrm{x}$ & $\mathrm{x}$ & $\mathrm{x}$ & $\mathrm{x}$ & $\mathrm{x} \quad 2$ & $\mathrm{x} \quad \mathrm{X}$ & $\mathrm{x} \quad \mathrm{x}$ & $\mathrm{x}$ & $\mathrm{x}$ & $\mathrm{x} \quad \mathrm{x}$ & $\mathrm{x} \quad \mathrm{X}$ & $\mathrm{x}$ & $\mathrm{x}$ & $\mathrm{X}$ & $\mathrm{x} \quad \mathrm{x}$ & $\mathrm{x}$ & $\mathrm{x}$ & $\mathrm{x} \quad \mathrm{X}$ & $\mathrm{x} \quad \mathrm{X}$ & 23 \\
\hline $\begin{array}{l}\text { Fatores de } \\
\text { desenvolvimento }\end{array}$ & $\mathrm{x}$ & $\mathrm{x}$ & $\mathrm{x}$ & $\mathrm{x}$ & $\mathrm{x}$ & $\mathrm{X} \quad \mathrm{x}$ & $\mathrm{x} \quad \mathrm{x}$ & $\mathrm{X} \quad \mathrm{X}$ & $\mathrm{x}$ & $\mathrm{x}$ & $\mathrm{x}$ & $\mathrm{X} \quad \mathrm{X}$ & $x$ & $\mathrm{x}$ & $\mathrm{x}$ & $\mathrm{X} \quad \mathrm{x}$ & $\mathrm{x}$ & $\mathrm{x}$ & $\mathrm{X} \quad \mathrm{X}$ & $\mathrm{x} \quad \mathrm{x}$ & 23 \\
\hline Fatores mecânicos & $\mathrm{x}$ & $\mathrm{x}$ & $\mathrm{x}$ & $\mathrm{x}$ & & $\mathrm{x}$ & & $\mathrm{X} \quad \mathrm{X}$ & $\mathrm{x}$ & $\mathrm{x}$ & $\mathrm{x}$ & $\mathrm{x}$ & $x$ & & $\mathrm{x}$ & $\mathrm{X} \quad \mathrm{x}$ & $\mathrm{x}$ & $\mathrm{x}$ & $\mathrm{X} \quad \mathrm{X}$ & $\mathrm{X} \quad \mathrm{X}$ & 19 \\
\hline Pigmentação alterada & $\mathrm{x}$ & $\mathrm{x}$ & $\mathrm{x}$ & $\mathrm{x}$ & & $\mathrm{x}$ & & $\mathrm{x} \quad \mathrm{x}$ & $\mathrm{x}$ & & $\mathrm{x} \quad \mathrm{x}$ & $\mathrm{x}$ & $\mathrm{x}$ & & $\mathrm{x}$ & $\mathrm{x} \quad \mathrm{x}$ & $\mathrm{x}$ & $\mathrm{x}$ & $\mathrm{x} \quad \mathrm{x}$ & $\mathrm{x} \quad \mathrm{x}$ & 18 \\
\hline Circulação alterada & & & $\mathrm{x}$ & $\mathrm{x}$ & $\mathrm{x}$ & & $\mathrm{x} \quad \mathrm{x}$ & $\mathrm{x} \quad \mathrm{x}$ & $\mathrm{x}$ & $\mathrm{x}$ & $x \quad x$ & $\mathrm{x} \quad \mathrm{x}$ & $x$ & $\mathrm{x}$ & & $\mathrm{x} \quad \mathrm{x}$ & $\mathrm{x}$ & $\mathrm{x}$ & $\mathrm{x}$ & $\mathrm{x}$ & 18 \\
\hline $\begin{array}{l}\text { Alteração do turgor da } \\
\text { pele }\end{array}$ & & $\mathrm{x}$ & $\mathrm{x}$ & $\mathrm{x}$ & $\mathrm{x}$ & & & $\mathrm{x} \quad \mathrm{x}$ & $\mathrm{x}$ & $\mathrm{x}$ & & & $\mathrm{x}$ & $\mathrm{x}$ & $\mathrm{x}$ & $\mathrm{x} \quad \mathrm{z}$ & $\mathrm{x}$ & $\mathrm{x}$ & $\mathrm{x} \quad \mathrm{x}$ & $\mathrm{x} \quad \mathrm{x}$ & 17 \\
\hline $\begin{array}{l}\text { Alteração do estado } \\
\text { nutricional }\end{array}$ & & $\mathrm{x}$ & & $\mathrm{x}$ & & $\mathrm{x} \quad \mathrm{x}$ & $\mathrm{x}$ & $\mathrm{x}$ & $\mathrm{x}$ & $\mathrm{x} \quad \mathrm{x}$ & $\mathrm{x}$ & $\mathrm{x}$ & $x$ & $\mathrm{x}$ & $\mathrm{x}$ & $\mathrm{x} \quad \mathrm{x}$ & $\mathrm{x}$ & & $\mathrm{x} \quad \mathrm{x}$ & $\mathrm{x} \quad \mathrm{x}$ & 17 \\
\hline $\begin{array}{l}\text { Força muscular/ } \\
\text { motricidade }\end{array}$ & & & $\mathrm{x}$ & $\mathrm{x}$ & & $\mathrm{x} \quad \mathrm{x}$ & $\mathrm{x} \quad \mathrm{x}$ & $\mathrm{x} \quad \mathrm{x}$ & $\mathrm{x}$ & & & $\mathrm{x} \quad \mathrm{x}$ & $x$ & $\mathrm{x}$ & $\mathrm{x}$ & & & $\mathrm{x}$ & $\mathrm{x} \quad \mathrm{x}$ & $\mathrm{x} \quad \mathrm{x}$ & 16 \\
\hline Umidade & $\mathrm{x}$ & & & $\mathrm{x}$ & & $\mathrm{X} \quad \mathrm{x}$ & $\mathrm{x}$ & & $\mathrm{x}$ & $\mathrm{x}$ & & $\mathrm{x}$ & $x$ & $\mathrm{x}$ & $\mathrm{x}$ & & & & $\mathrm{x} \quad \mathrm{x}$ & $\mathrm{x}$ & 12 \\
\hline Extremos de temperatura & & $\mathrm{x}$ & $\mathrm{x}$ & $\mathrm{x}$ & $\mathrm{x}$ & $\mathrm{x}$ & & & & & $\mathrm{x}$ & $\mathrm{x}$ & $x$ & & $\mathrm{x}$ & & $\mathrm{x}$ & $\mathrm{x}$ & & $\mathrm{x}$ & 11 \\
\hline Fatores cognitivos & & & $\mathrm{x}$ & & $\mathrm{x}$ & $\mathrm{x} \quad \mathrm{x}$ & $\mathrm{x}$ & $\mathrm{x}$ & $\mathrm{x}$ & $\mathrm{x} \quad \mathrm{x}$ & $\mathrm{x}$ & & & $\mathrm{x}$ & $\mathrm{x}$ & & & $\mathrm{x}$ & & $\mathrm{x}$ & 12 \\
\hline Fatores psicogênicos & & $\mathrm{x}$ & & & & $\mathrm{x} \quad \mathrm{x}$ & $\mathrm{x} \quad \mathrm{x}$ & $\mathrm{x} \quad \mathrm{x}$ & $\mathrm{x}$ & & $\mathrm{x} \quad \mathrm{x}$ & $\mathrm{x}$ & & $\mathrm{x}$ & $\mathrm{x}$ & & & & & $\mathrm{x}$ & 11 \\
\hline Substância química & $\mathrm{x}$ & & & $\mathrm{x}$ & & $\mathrm{x} \quad \mathrm{x}$ & $\mathrm{x}$ & & & $\mathrm{x}$ & & & $\mathrm{x}$ & $\mathrm{x}$ & & & & & $\mathrm{X} \quad \mathrm{X}$ & $\mathrm{x}$ & 09 \\
\hline Sensibilidade alterada & & & $\mathrm{x}$ & $\mathrm{x}$ & $\mathrm{x}$ & & & $\mathrm{x} \quad \mathrm{x}$ & & & & $\mathrm{x}$ & $\mathrm{x}$ & $\mathrm{x}$ & & & & & & $\mathrm{x}$ & 08 \\
\hline Proeminências ósseas & & $\mathrm{x}$ & $\mathrm{x}$ & & & & & & & $\mathrm{x} \quad \mathrm{x}$ & $\mathrm{x} \quad \mathrm{x}$ & $\mathrm{x}$ & & & & & & & $\mathrm{x}$ & $\mathrm{x}$ & 07 \\
\hline Alteração metabólica & & & & & & & $\mathrm{x}$ & $\mathrm{x}$ & $\mathrm{x}$ & & & & $\mathrm{x}$ & & & $\mathrm{x}$ & & $\mathrm{x}$ & & $\mathrm{x}$ & 06 \\
\hline Imobilização física & & & & & & & $\mathrm{x}$ & $x$ & & & & $\mathrm{x}$ & $x$ & & & & & & & $\mathrm{x}$ & 03 \\
\hline Total & 09 & 12 & 14 & 15 & 10 & 141 & 1214 & 414 & 15 & 131 & 121 & 1113 & $\begin{array}{ll}3 & 14\end{array}$ & 14 & 14 & 111 & 111 & 131 & & 1515 & 299 \\
\hline $\begin{array}{l}\text { Pontuação para Escala } \\
\text { de Waterlow }\end{array}$ & 06 & 11 & 08 & 19 & 10 & 121 & 1533 & 319 & 26 & 142 & 211 & 1713 & 316 & 18 & 18 & 100 & 081 & 131 & $182 \varepsilon$ & 2822 & 375 \\
\hline
\end{tabular}


A luz solar é a principal fonte de radiação que nos envolve. $\mathrm{O}$ efeito fotobiológico cumulativo de exposição à radiação solar afeta especialmente as peles mais claras, levando à atrofia da pele, proeminência e aumento da pigmentação dos sulcos, além das alterações morfológicas correspondentes, ou seja, as fotodermatoses tóxicas primárias ${ }^{(1)}$.

A amostra foi composta, quanto à raça autoreferida, de $16(69,56 \%)$ idosos brancos, três $(13,05 \%)$ negros e quatro $(17,39 \%)$ pardos, nenhum relatou uso atual e/ou anterior de filtro ou protetor solar.

Fatores imunológicos foram atribuídos à todos os idosos da amostra, pois, segundo estudiosos da área ${ }^{(1-2,11)}$, com aumento da idade, há um declínio da atividade imunológica ${ }^{(2)}$. A literatura descreve duas explicações para isto. A primeira é a diminuição fisiológica das células de Langerhans da pele no processo de envelhecimento ${ }^{(1)}$. A segunda é a involução e atrofia fisiológica do timo, nos primeiros 50 anos de vida humana. Com isto, acredita-se haver influência na atividade dos linfócitos timo-dependentes ${ }^{(11)}$.

O fator mecânico foi encontrado em 19 idosos $(82,61 \%)$. Dezesseis $(69,56 \%)$ faziam uso de esparadrapos comuns para fixação do dispositivo para infusão venosa, enquanto dois $(8,70 \%)$ usavam micropore, além de ataduras. Um participante (4,35\%) fazia uso de bolsa de colostomia. Estes dispositivos possuem ação aderente à pele, o que favorece o rompimento da integridade cutânea, a partir da sua manipulação e especialmente na remoção.

Dos participantes, 17 (73,91\%) tinham o turgor da pele diminuído. É esperado o retorno delongado da prega cutânea, em idosos, de forma proporcional à idade, como conseqüência da redução do entrançamento dos ramos de fibras elásticas em torno dos feixes de colágeno na derme, além da falência do aparelho elástico, devido à possível deficiência no turn over do colágeno, com o avançar da idade ${ }^{(12)}$.

Em 17 idosos (73,91\%) encontrou-se alterações de pigmentação relacionadas à punção venosa, como equimoses e hematomas. A dificuldade de acesso venoso que os idosos apresentam ocorre principalmente devido à fragilidade cutânea, às veias escleróticas e os distúrbios circulatórios, que requerem uma maior atenção na punção e manutenção desses acessos ${ }^{(13)}$.

Além das alterações na pigmentação relativas à punção venosa, 18 idosos $(78,26 \%)$ apresentavam manchas hipercrômicas e/ou hipocrômicas de tamanhos diversos, em vários segmentos corpóreos e com variação de únicas a difusas. Assim, 18 (78,26\%), possuíam manchas hipercrômicas, 11 (47,83\%) apresentavam manchas hipocrômicas e quatro (17,39\%) hiperemia, reativa a remoção de pressão.

O surgimento de manchas com o passar dos anos, característica do envelhecimento, é citado por autores ${ }^{(1-}$ ${ }^{2,12)}$. Em idosos ocorre desorganização e achatamento no arranjo das células basais, com acúmulo menor de melanossomas e menor densidade de melanócitos decorrentes de alterações estruturais e resultantes de fatores extrínsecos como a radiação solar ${ }^{(12)}$.

Dos idosos pesquisados 17 (73,91\%) apresentaram estado nutricional propício ao aumento do risco de prejuízo da integridade cutânea. As refeições anteriores à internação, em seus domicílios, eram, regularmente, arroz e feijão, em todos os casos; acrescida de carne em $60,87 \%$, e verduras e/ou legumes para 43,48\%. Ainda, 14 idosos (60,87\%) mencionaram diminuição no apetite, aceitando parcialmente a dieta oferecida no hospital, não ingerindo ou ingerindo minimamente alimentos. Os índices de massa corporal (IMC) variaram entre 16,35 e $30,46 \mathrm{~kg} / \mathrm{m}^{2}$, sendo que 9 idosos $(39,13 \%)$ apresentavam baixo peso (IMC $<20$ $\mathrm{kg} / \mathrm{m}^{2}$ ), 9 (39,13\%) dentro da faixa de normalidade (IMC = 20 a 24,99 kg/m²), 4 (17,39\%) em sobrepeso (25 a $\left.29,99 \mathrm{~kg} / \mathrm{m}^{2}\right)$ e um $(4,35 \%)$ obeso $\left(30 \text { a } 39,99 \mathrm{~kg} / \mathrm{m}^{2}\right)^{(14)}$. A maioria dos idosos (82,61\%) referiu perda de peso recente, 11 (47,83\%) não souberam especificar o quanto. Em dois (8,70\%) foi verificada perda de dez quilogramas ou mais em período inferior a um mês.

Diversos fatores podem influenciar na má nutrição do idoso, como problemas mecânicos relacionados à mastigação e à digestão, além de aspectos socioeconômicos, que restringem o acesso aos alimentos "nobres"(11). As doenças crônicas podem influenciar no apetite e nas necessidades metabólicas, desfavorencendo as condições de nutrição.

Seis idosos (26,09\%) apresentaram "alteração metabólica”, constatada por registros médicos de alterações do sistema endócrino, como diabetes mellitus (DM), hipo e hipertireodismo. Estas doenças contribuem, direta e/ou indiretamente, para a fragilidade cutânea. O hipotireoidismo leva o indivíduo a apresentar pele áspera e ressecada, devido a secreção diminuída das glândulas sebáceas e sudoríparas, além de hipotermia resultante da vasoconstrição cutânea. No hipertireoidismo, os sintomas mais comuns não influenciam tão diretamente a pele, mas predispõem à condições clínicas como emagrecimento, fraqueza, alterações cardiovasculares e mentais, que por sua vez são favoráveis ao risco de prejuízo na integridade cutânea. O DM contribui para o 
risco de desenvolvimento de lesões, à medida que as complicações decorrentes da progressão dessa afecção surgem. A neuropatia autonômica, nas suas várias condições, desencadeia a anidrose dos membros, perda da sensação dolorosa e de pressão, bem como alterações no formato dos pés, predispondo o indivíduo ao risco em questão. Por outro lado, o próprio processo de envelhecimento favorece alterações metabólicas, que culminam em modificações fisiológicas, as quais variam entre os indivíduos, não sendo possível estabelecer a idade em que se inicia todo este processo ${ }^{(11)}$.

Em 100\% dos idosos foi verificado o fator de risco "medicamentos". Todos usavam pelo menos um composto que apresentava possibilidades de reações adversas dermatológicas: erupções cutâneas, prurido e fotossensibilidade. Além disso, a quantidade de medicamentos utilizados, pelos idosos era relativamente alta, sendo relatados casos de até 16 medicamentos concomitantes no domicilio, e 13 na atual internação.

Fatores cognitivos e psicogêncios podem interferir na possibilidade do idoso desenvolver alguma lesão cutânea, à medida que a diminuição do desempenho intelectual, memória e capacidade de resolução de problemas e percepção podem afetar o desempenho nas atividades cotidianas que requeiram iniciativa, planejamento e avaliação de comportamentos, contribuindo para limitação da capacidade de proteção e cuidado da própria pele.

Dos idosos, 11 (47,83\%) apresentaram fatores psicogênicos, sendo que 9 (39,13\%) manifestaram indícios depressivos captados pela escala utilizada. Dois (8,7\%), demonstraram feição e comportamento evidenciando tristeza, desânimo, pesar e/ou irritação. Ainda, 12 idosos $(52,17 \%)$ tiveram pontuação correspondente ao dano intelectual, sendo 5 (41,66\%) com “dano leve”, 6 (50\%), "moderado”, e um (8,34\%), "grave”, sendo o último na faixa etária de 80 e 89 anos.

Encontrou-se que 16 idosos (69,56\%) apresentavam força muscular e/ou motricidade prejudicadas, sendo que, de toda a amostra, 14 (60,87\%) eram independentes para deambular, 4 $(17,39 \%)$ necessitavam de ajuda de uma pessoa, 2 (8,70\%) de algum mecanismo auxiliar, como suporte de soro e $3(13,04 \%)$ se encontravam impossibilitados de deambular, mas movimentando ativamente no leito.

De uma forma geral, com o envelhecimento, há um declínio da área motora resultante do próprio processo fisiológico, como também, da falta de atividade física regular, sensações dolorosas e hábitos de vida ${ }^{(15)}$. Por outro lado, a internação hospitalar predispõe o idoso a declínio funcional, podendo resultar em agravos no seu estado geral e na sua qualidade de vida ${ }^{(16)}$.

Dos idosos, 18 (78,26\%) apresentaram “circulação alterada”. Conforme registros obtidos nos prontuários, 78,26\% dos idosos apresentavam hipertensão arterial sistêmica, 52,17\% tinham cardiopatia, $39,13 \%$ apresentavam problemas vasculares (obstrução arterial crônica). Verificou-se ainda que 8 idosos (34,78\%) apresentavam resultados laboratoriais de hematócrito menores que o limite inferior da normalidade; da mesma forma os resultados de hemoglobina foram baixos em 6 participantes (26,09\%). Também, 15 idosos (83,34\%) apresentaram perfusão periférica diminuída, sendo $11(73,33 \%)$ em ambos os membros inferiores, um (6,67\%) em um dos membros inferiores e 3 (20\%) em todos os membros.

Em 8 idosos (34,78\%) foi identificada “sensibilidade alterada”, dos quais, 5 (62,50\%) com duas ou mais alterações; 2 (25\%) exibiram alterações na sensibilidade tátil e um (12,50\%) na sensibilidade dolorosa. A pele apresenta corpúsculos nervosos espalhados nas diferentes regiões corpóreas e com capacidade de distinções sensitivas diversas. Com o envelhecimento, diminui a quantidade e o volume de alguns desses corpúsculos, como os de Vater-Pacini, responsáveis pela sensibilidade tátil e de pressão ${ }^{(1)}$.

Houve, entre os idosos, 14 menções (60,86\%) de dor, dentre elas, em região abdominal, cefaléia e membros inferiores. Considerando que a dor é definida como uma sensação indicativa de uma agressão real e/ou iminente, e que ao mesmo tempo a atenção e consciência/ percepção podem ser alterados com sua presença, pode-se inferir que a ocorrência de sensações dolorosas, adicionadas às anormalidades constatadas na sensibilidade, entre os participantes, também os expunha a risco de alteração na integridade cutânea, visto que a existência de dor em determinada região do corpo poderia “desviar” a percepção dolorosa proveniente de possíveis estímulos agressores, em outros locais, interferindo assim na capacidade de proteção exercida pela pele ${ }^{(17)}$.

Encontrou-se que 7 idosos (30,43\%) possuíam proeminências ósseas salientes, sendo 2 em mais de quatro regiões corpóreas, considerando ossos dos membros e pélvicos. Estes idosos também apresentavam baixo peso (Tabela 1).

Verificou-se 12 idosos (52,17\%) que estavam em condições que favoreciam e/ou aumentavam a ocorrência de umidade na pele, como leito e/ou vestes úmidos com sudorese ou sujidade ou sangue, uso de 
fraldas descartáveis, presença de colostomia, incontinência urinária e/ou intestinal ocasional. Em 9 $(39,13 \%)$ dos idosos esta umidade configurou-se também como o fator de risco "substância química", sendo que 5 tinham contato com fezes e urina, devido o uso de fraldas descartáveis, devido à incontinência urinária ou intestinal. Estas substâncias apresentam $\mathrm{pH}$ e compostos agressivos à pele, assim, é importante a ação de enfermagem no que se refere à promoção e manutenção da higiene, visto a real possibilidade de complicações, especificamente lesões perineais ${ }^{(18)}$.

O contato com "extremos de temperatura" foi verificado em 11 idosos (47,83\%), que referiram sensação térmica desconfortável, quente e/ou fria, nas últimas 48 horas do dia da coleta de dados. Além disso, dois deles estavam em hipertermia no momento da entrevista, e três referiram aplicação de frio ou calor no mesmo período.

O risco para desenvolvimento para UP mostrouse presente em 18 (78,26\%) idosos, sendo o escore alto em 8 participantes (34,78\%) e altíssimo em 5 (21,74\%). $\mathrm{O}$ fato do idoso não apresentar risco detectado, pela escala para desenvolvimento de UP, não os isenta de apresentar outros tipos de lesões. Pode-se observar pela Tabela 1, que os três idosos considerados "sem risco" para UP, apresentaram fatores de risco para integridade de pele prejudicada, segundo o referencial adotado ${ }^{(5)}$.

Na condição da hospitalização, com a saúde debilitada, os idosos estão em situação de alto risco em relação ao rompimento de sua pele, devido à maior probabilidade de contato dos agentes agressores e maior poder lesivo dos mesmos nesta faixa etária pelo comprometimento das reservas fisiológicas de proteção.

Fatores de risco identificados como: estado nutricional alterado, exposição à radiação solar, fatores imunológicos e alterações metabólicas ligadas à doenças crônicas não transmissíveis, podem ser evitados adotando-se práticas saudáveis desde a mais tenra idade, o que poderia ser mais estimulado mediante a implantação de políticas públicas. A Política Nacional de Saúde da Pessoa Idosa ${ }^{(19)}$ considera, por meio da Portaria $n^{\circ}$. 2.528/06, dentre outros aspectos, a necessidade de um investimento da sociedade, e em especial os profissionais da área de saúde, ao envelhecimento ativo e saudável da população brasileira, compreendendo ações de prevenção e promoção de saúde aos indivíduos dessa faixa etária.

Alguns FR identificados neste estudo como umidade e fatores mecânicos, podem ser minimizados, no ambiente hospitalar, se houver direcionamento institucional no tocante a um atendimento de qualidade. A manutenção da higiene corporal, especialmente higiene íntima, as trocas mais freqüentes de lençóis e fraldas, o controle de um ambiente agradável em relação à temperatura, bem como o uso de fitas adesivas menos agressivas à pele, são cuidados de enfermagem contribuidores diretos para a redução da ação desses fatores. Porém, estas ações, até certo ponto, são dependentes das condições oferecidas pelas instituições hospitalares, no que se referem aos recursos humanos e materiais.

Para um atendimento humanizado, holístico e de qualidade, é necessário o rompimento da estrutura burocrática de organização das instituições hospitalares, remetendo necessariamente ao questionamento da situação das tecnologias de gestão ou modos de organização do trabalho, em especial de enfermagem, em vigor na maioria das instituições ${ }^{(20-21)}$.

\section{CONSIDERAÇÕES FINAIS}

Os resultados obtidos nesta pesquisa apontam que $100 \%$ dos idosos hospitalizados apresentaram risco de desenvolverem integridade de pele prejudicada. Entre os fatores de risco (FR) identificados, alguns representam as conseqüências do processo de senescência, outros denotam fragilidade ou envelhecimento mal sucedido, e outros são específicos do contexto da atenção hospitalar. Aqueles resultantes do processo de envelhecimento, saudável ou não, poderiam ser minimizados ou prevenidos no âmbito de políticas públicas de promoção da saúde. Os concernentes à hospitalização podem ser manejados mediante o planejamento de assistência individualizada, resolutiva e de qualidade.

É necessário que as instituições e os profissionais de saúde repensem o atendimento a esta população, e busquem ações que contribuam para a melhoria e eficácia da assistência. Essas ações podem ser dispendiosas em princípio, mas certamente menos onerosas em comparação àquelas inerentes às complicações desencadeadas por uma lesão de pele, em um idoso hospitalizado.

A elaboração de um protocolo de enfermagem referente à abordagem e seguimento da população idosa que é admitida nas clínicas da instituição hospitalar poderia contribuir substancialmente na sistematização da assistência de enfermagem (SAE). Em conseqüência, a qualidade da assistência poderia ser aprimorada e a visibilidade dos resultados das 
intervenções de enfermagem, aumentada.

É notória a dificuldade existente para a enfermagem ser exercida em sua plenitude, no interior da estrutura rígida burocrática das instituições hospitalares, ainda pautada na modalidade de trabalho funcionalista, exercendo exigências e condicionando a equipe de enfermagem, de forma especial, enfermeiros, à tarefas rotineiras e estáticas. Contudo, mediante a crescente exigência de qualidade da assistência à saúde das pessoas, bem como desenvolvimento de tecnologias nesta área, vislumbrase a possibilidade de transformação desta realidade, colocando para estes profissionais o desafio de buscar maior autonomia e responsabilização pelo atendimento das necessidades de cuidado dos indivíduos.

São fatores limitantes deste estudo o tamanho da amostra e a amostragem não probablilística, o que não permite generalização dos resultados. Porém, dada a magnitude e a natureza do desfecho identificado, esta pesquisa indica a ocorrência deste fenômeno na população idosa hospitalizada, sinalizando a necessidade de outros estudos em diferentes contextos hospitalares, para maior conhecimento nesta área, e melhor compreensão do DE em estudo, inerentes aos idosos, proporcionando subsídios para o planejamento da assistência de enfermagem a esses indivíduos.

\section{REFERÊNCIAS}

1. Azulay RD; Azulay DR, Dermatologia. $2^{\mathrm{a}}$ ed. Rio de Janeiro: Guanabara Koogan; 1999.

2. Carvalho Filho ET. Fisiologia do envelhecimento. In: Papaléo Netto M. Gerontologia. São Paulo: Atheneu; 1996. p. 60-72.

3. Alvarenga MRM, Mendes MMR. O perfil das readmissões de idosos num hospital geral de Marília SP. Rev Latino-Am Enferm. 2003 Mai/Jun;11(3):305-11.

4. Araújo LAO, Santana RF, Bachion MM. Mobilidade física prejudicada em idosos: fatores relacionados e características definidoras. Rev Bras Enferm. 2002 Jan/ Fev;55(1):19-25.

5. North American Nursing Diagnosis Association. Diagnóstico de enfermagem da NANDA: definições e classificação - 2005-2006. Porto Alegre: Artmed; 2006.

6. Rocha ABL, Barros SMO. Tradução para a língua portuguesa, adaptação transcultural e aplicação clínica da escala de Waterlow para avaliação de risco de desenvolvimento de úlcera de decúbito. Estima. 2005 Abr/Jun;3(2):43-44.
7. IBGE. Perfil dos idosos responsáveis pelos domicílios no Brasil, 2000. Rio de Janeiro; 2002.

8. Gonçalves LTT. Tendências assistenciais de enfermagem -assistência ao idoso. In: Anais do $51^{\circ}$ Congresso Brasileiro de Enfermagem; Florianópolis, 1999.

9. Maia FOM, Duarte YAO, Lebrão ML. Análise dos óbitos em idosos no estudo SABE. Rev Esc Enferm USP. 2006;40(4):540-7.

10. Araújo LAO, Bachion MM. Diagnósticos de enfermagem do Padrão Mover em idosos de uma comunidade atendida pelo Programa Saúde da Família. Rev Esc Enferm USP. 2005;39(1):53-61.

11. Carvalho Filho ET, Papaléo Netto, M. Geriatria: fundamentos, clínica e terapêutica São Paulo: Atheneu; 2000.

12. Oriá RB, Ferreira FVA, Santana EN, Fernandes MR, Brito GAC. Estudo das alterações relacionadas com a idade na pele humana, utilizando métodos de histomorfometria e autofluorescência. Anais Brasileiros de Dermatologia. 2003 Jul/Ago;78(4):425-34.

13. Ferreira NMLA, Marassi RP. Avaliando condutas na preservação da infusão venosa no doente hospitalizado. Rev Prática Hospitalar. 2005 Mai/Jun;39(7).

14. Najas MS, Sachs A. Avaliação nutricional do idoso. In: Papaléo Netto M. Gerontologia. São Paulo: Atheneu; 1996. p. 242-61.

15. Cantera IR, Domingo PL. Guia prático de enfermagem: geriatria. Rio de Janeiro: McGraw; 2000.

16. Gorzoni ML, Pires SL. Idosos asilados em hospitais gerais. Rev Saúde Públ. 2006; 40(6):1124-30.

17. Macbryde, CM, Blacklow RS. Sinais e sintomas: fisiopatologia aplicada e interpretação clínica. $5^{\mathrm{a}}$ ed. Rio de Janeiro: Guanabara Koogan; 1975.

18. Rodrigues RAP, Mendes MMR. Incontinência urinária em idosos: proposta para conduta da enfermeira. Rev Latino-Am Enferm. 1994 Jul; 2(2):5-20.

19. Ministério da Saúde (BR). Política Nacional de Saúde da Pessoa Idosa. Portaria 1395/GM de 10 de dezembro de 1999. Brasília; 1999.

20. Silva TG, Madureira VSF, Trentini M. Processo de ensino-aprendizagem para implantação do diagnóstico de enfermagem em unidade de terapia intensiva. Cogitare Enferm. 2007 Jul/Ago;12(3):279-86.

21. Pavanati KCA, Erdmann AL. Intercorrências no trabalho de uma equipe de enfermagem em unidade de internação médica. Cogitare Enferm. 2006 Jan/Abr; 11(1):24-30. 


\section{Anexo}

Síntese dos procedimentos adotados para avaliação dos fatores de risco do diagnóstico de risco de integridade de pele prejudicada.

- Radiação: avaliação de história de exposição à radiação solar, no período de 10 a 16 horas, e uso de filtro solar; investigação de história de exposição a raios-X ou radioterapia.

- Imobilização física: verificação da presença de alguma imobilização física na internação atual.

- Fatores mecânicos: investigação sobre contato com esparadrapo, micropore ou outro dispositivo que desempenhe ação mecânica sobre a pele.

- Extremos de temperatura: avaliação de história de aplicação de frio ou calor, nas últimas 48 horas; pesquisa da história de temperatura ambiente e/ou sensação desconfortável de frio ou calor, nas últimas 48 horas; verificação da temperatura axilar, no momento da entrevista, e resultados registrados nas últimas 48 horas.

- Umidade: avaliação de condições do leito e vestes quanto à presença de urina, fezes, sudorese, sujidade, sangue e/ou outra secreção; verificação do uso de sonda vesical de demora, condom e fralda descartável.

- Substância química: investigação acerca do contato com fezes, urina ou outro fluido biológico; investigação de história de alergia a esparadrapo, soluções com iodo ou outra substância.

- Extremos de idade: investigação da data de nascimento e idade, no dia da avaliação, em anos e meses.

- Medicamentos: investigação sobre o uso de medicamentos no domicilio e na internação atual.

- Fatores de desenvolvimento: como este estudo avaliou indivíduos na fase de desenvolvimento da velhice, buscou-se na literatura todos os aspectos relacionados à integridade da pele nesta fase de desenvolvimento humano.

- Fatores imunológicos: investigação de história de doenças crônicas não transmissíveis; identificação do motivo da internação atual; avaliação do resultado da contagem diferencial de leucócitos.

- Proeminências ósseas: Investigação de história de perda de peso recente; avaliação de presença de proeminência óssea.

- Sensibilidade alterada: questionamento da presença e características de dor, formigamento e/ou adormecimento; aplicação do teste de sensibilidade para dor e temperatura, posição, discriminação e tátil fina.

- Pigmentação alterada: verificação de presença de alterações na coloração e relevo da pele, a partir do uso de uma escala de cor, para auxílio na estimativa das possíveis variações nos segmentos corpóreos, bem como a investigação de possível associação com punções venosas e/ou arteriais e traumatismo.

- Alteração metabólica: avaliação de histórico de doença (s) pré-existente (s) relacionada (s) ao sistema endócrino: diabetes mellitus, hiper ou hipotireoidismo.

- Circulação alterada: avaliação de história de cardiopatia ou doença relacionada ao sistema circulatório; avaliação da perfusão periférica.

- Alterações no turgor da pele: exame das condições da pele quanto à presença de ressecamento, oleosidade, sudorese, aspereza, edema e turgor.

- Alterações no estado nutricional: realização de medidas antropométricas (peso, altura) e cálculo de índice de Quetelet, para avaliação do índice de massa corporal; avaliação de resultados de estudos bioquímicos do sangue (hematócrito e hemoglobina); investigação quanti e qualitativa da alimentação do idoso anteriormente à internação atual; avaliação do apetite atual e aceitação da dieta no hospital.

- Fatores cognitivos: aplicação da escala Mini Mental de Pfeiffer.

- Fatores psicogênicos: observação da aparência ou expressões de tristeza, irritação, apatia entre outros; aplicação da escala de depressão geriátrica de Yessavage.

- Integridade da musculatura e motricidade: exame das condições de mobilidade; exame da força muscular.

Cogitare Enferm 2008 Jul/Set; 13(3):428-36 